\title{
ON WU'S FORM OF THE FIRST MAIN THEOREM OF VALUE DISTRIBUTION
}

JOHN J. HIRSCHFELDER

In the First Main Theorem of value distribution for holomorphic maps into a compact Kähler manifold $A$, the central object is a differential form $\lambda_{a}$ on $A$ with a singularity at $a \in A$. The most satisfactory $\lambda_{a}$ was given by $\mathrm{Wu}$ in [5]. His construction, using the Green function, has the advantage that $\lambda_{a}$ is automatically invariant under the group of isometries of $A$; more precisely, if $i: A \rightarrow A$ is an isometry with $i(a)=b$, then $i^{*} \lambda_{b}=\lambda_{a}$. This property is difficult to obtain with any other construction.

In this note we apply the technical machinery developed by Stoll and the author [1], [2], [3], [4] to Wu's $\lambda_{a}$ to show that it is suitable for value distribution theory in arbitrary fiber dimension. We also show that the terms of the First Main Theorem are continuous functions.

$\nu_{f}$ will denote the multiplicity of the holomorphic map $f$; see [2]. $O_{k}$ will denote any differential form of degree $k$ whose coefficients are locally bounded; the subscript will usually be omitted. Of course it will never mean the same thing twice. On $C^{n}$ set $v_{1}=(i / 2) \Sigma_{\mu=1}^{n} d z_{\mu}$ $\wedge d \bar{z}_{\mu}$ and $v_{1}^{k}=v^{k} / k !$.

If $M$ is a complex manifold and $H$ an open subset of $M$, then $S=\bar{H}-H$ is a boundary manifold of $H$, provided that for every point of $S$ there is a $C^{\infty}$ coordinate system $\left(x_{1}, \cdots, x_{2 n}\right)$ valid in a neighborhood $U$ of the point, such that in $U, S$ is described by $x_{1}=0$ and $H$ by $x_{1}<0 . S$ will be considered as oriented to the exterior of $H$.

1. We collect the technical results which we will need.

LeMma 1. Let $k \geqq 0,0 \leqq s \leqq n$ and $0 \leqq t \leqq n$ be integers such that $s+t<2 n$. Let $\phi(w, z)$ be a differential form of bidegree $(s, t)$ in $w \in C^{n}$, dependent on a parameter $z \in C^{n}$, whose coefficients are measurable and locally bounded. Let $M$ be a complex manifold, and let $f: M \rightarrow C^{n}$ be an open holomorphic map. Let $K$ be a compact subset of $M$. Let $\chi(w, z)$ be a differential form in $w \in M$ dependent also on a parameter $z \in C^{n}$, whose coefficients are measurable and locally bounded; then

$$
\begin{aligned}
F(z)= & \int_{w \in K}\left[\log (1 /|f(w)-z|]^{k}|f(w)-z|^{-(s+t)}\right. \\
& \cdot f^{*} \phi(w, z) \wedge \chi(w, z)
\end{aligned}
$$

Received by the editors April 29, 1969. 
exists for each $z \in C^{n}$, and is a measurable and locally bounded function on $\boldsymbol{C}^{n}$. If moreover $\boldsymbol{\phi}$ is continuous off the diagonal and $\chi$ is continuous off $\{(w, z) \mid f(w)=z\}$, then $F$ is continuous.

This is a restatement of Lemma 2.6 of [1].

Choose a function $h$ of class $C^{\infty}$ on $R$ such that $0 \leqq h(x) \leqq 1$ for all $x, h(x)=0$ if $x \leqq 0$, and $h(x)=1$ if $x \geqq 1$. A constant $B>0$ exists such that $2\left|h^{\prime}(x)\right| \leqq B$ for all $x$.

For $\rho>0$ define

$$
g_{\rho}(X)=h((2 X-\rho) / \rho) .
$$

Then

(a) $0 \leqq g_{\rho}(X) \leqq 1$,

(b) $X\left|g_{\rho}^{\prime}(X)\right| \leqq B$,

(c) $X \leqq \rho / 2 \Rightarrow g_{\rho}(X)=0$,

(d) $X \geqq \rho \Rightarrow g_{\rho}(X)=1$.

Now let $f: M \rightarrow C^{n}$ be a holomorphic map. Define $\gamma_{\rho}$ on $M$ by $\gamma_{\rho}(w)$ $=g_{\rho}(|f(w)|)$. Then $d \gamma_{\rho}=g_{\rho}^{\prime}(|f|)((d f \mid f)+(f \mid d f)) / 2|f|$. Set $h_{\rho}$ $=|f| g_{\rho}^{\prime}(|f|)$ and $\eta=((d f \mid f)+(f \mid d f)) / 2|f|$. Then the functions $h_{\rho}$ are uniformly bounded, $\eta$ is a locally bounded form, and

$$
d \gamma_{\rho}=h_{\rho} \eta /|f| \text {. }
$$

Define $L_{\rho}=\left\{w \in K \mid \rho / 2 \leqq \gamma_{\rho}(w) \leqq \rho\right\}$.

LeMma 2. Hypotheses as in Lemma 1, let also a measurable function $h_{\rho}$ be given for each $\rho>0$, and suppose that these functions are uniformly bounded on $K$. Then

$$
\lim _{\rho \rightarrow 0} \int_{L_{\rho}} h_{\rho}[\log (1 /|f|)]^{k}|f|^{-(s+t)} f^{*} \phi \wedge \chi=0 .
$$

See Stoll [4, Lemma 1.7].

Lemma 3. Hypotheses as above, let $\phi$ be a locally bounded form of degree $2 n-1$ on $\mathbf{C}^{n}$. Then

$$
\lim _{\rho \rightarrow 0} \int_{K} d \gamma_{\rho} \wedge|f|^{2-2 n}[\log (1 /|f|)]^{k} f^{*} \phi \wedge \chi=0 .
$$

Proof. The expression under the integral sign is

$$
-\left(h_{\rho} /|f|\right)|f|^{2-2 n}[\log (1 /|f|)]^{k} f^{*} \phi \wedge(\eta \wedge \chi) .
$$

Now decompose $\phi$ into forms of bidegrees $(n, n-1)$ and $(n-1, n)$ and apply Lemma 2 twice. q.e.d. 
Lemma 4. Hypotheses as above, and suppose also that $H$ is an open subset of $M$ such that $\bar{H}=K$ and that such that $S=\bar{H}-H$ is either empty or a smooth boundary manifold of $H$. Let $T$ be the support of $\chi$ on $S$ and suppose that $T \cap f^{-1}(0)$ is of measure zero on $f^{-1}(0)$. Then

$$
\begin{array}{r}
\lim _{\rho \rightarrow 0} \int_{H} d \gamma_{\rho} \wedge d^{c} \log (1 /|f|) \wedge|f|^{2-2 n f *^{*} v_{n}} \wedge \chi \\
=-\left[2 \pi^{n} /(n-1) !\right] \int_{H \cap f^{-1}(0)} \nu_{f} \chi .
\end{array}
$$

See Stoll [4, Lemma 1.8].

2. Let $A$ be a compact Kähler manifold of dimension $n$. Denote its fundamental form by $\omega_{1}$, and set $\omega_{k}=\omega_{1}^{k} / k !, \omega=\omega_{n}$. We assume that the metric is normalized so that $\int_{A} \omega=1$. Denote by $r(z, w)$ the distance between $z$ and $w$.

Denote by $g(z, w)$ the Green function on $A \times A$. Then

$$
\begin{aligned}
& g(z, w)=\frac{1}{4}(n-2) ! \pi^{-n} r(z, w)^{2-2 n}+r(z, w)^{3-2 n} O(z, w), \quad n>1, \\
& g(z, w)=(1 / 2 \pi) \log (1 / r(z, w))+O(z, w), \quad n=1 .
\end{aligned}
$$

There exists a constant $C$ such that $g(z, w)+C$ is everywhere positive.

Define

$$
\lambda_{z}(w)=(g(z, w)+C) \omega_{n-1}(w) .
$$

Then $d d^{c} \lambda_{z}=\omega$. (See Wu [5, I and II ].)

Let $a \in A$, and let $\left(z_{1}, \cdots, z_{n}\right)$ be a holomorphic coordinate system in a neighborhood $U$ centered at $a$, such that in the underlying real coordinate system the metric tensor at $a$ is represented by the identity matrix. Set

$$
B(z, w)=|z-w| / r(z, w)
$$

where $|z-w|$ is computed in the $\left(z_{1}, \cdots z_{n}\right)$ coordinate system. $B$ is continuous and positive on $U \times U$ and $B(a, a)=1$.

From (1), (2), and (3) we see

$$
\begin{aligned}
& \lambda_{z}(w)=|z-w|^{2-2 n} O(z, w) v_{n-1}, \quad n>1, \\
& \lambda_{z}(w)=(1 / 2 \pi) \log (1 /|z-w|)+O(z, w), \quad n=1 .
\end{aligned}
$$

From (1), (see $\mathrm{Wu}[\mathbf{5}, \mathrm{II}, \S 8]$ for justification) 


$$
\begin{aligned}
d_{w}^{c} \lambda_{z}(w)= & {\left[-\left[(n-1) ! / 2 \pi^{n}\right] r(z, w)^{1-2 n} d_{w}^{c} r(z, w)\right.} \\
& \left.+O_{1}(z, w) r(z, w)^{2-2 n}\right] \wedge \omega_{n-1}(w) \quad(\text { if } n>1) \\
= & {\left[\left[(n-1) ! / 2 \pi^{n}\right] r(z, w)^{2-2 n} d_{w}^{c} \log (1 / r(z, w))\right.} \\
& \left.+O_{1}(z, w) r(z, w)^{2-2 n}\right] \wedge \omega_{n-1}(w) \quad(\text { any } n) .
\end{aligned}
$$

Now $\log (1 / r(z, w))=\log B(z, w)+\log (1 /|z-w|)$ and $\log B(z, w)$ is locally bounded; hence

$$
\begin{aligned}
d_{w}^{c} \lambda_{z}(w)= & {\left[\left[(n-1) ! / 2 \pi^{n}\right] B(z, w)^{2 n-2} r(z, w)^{2-2 n} d_{w}^{c} \log (1 /|z-w|)\right.} \\
& \left.+O_{1}(z, w)|z-w|^{2-2 n}\right] \wedge \omega_{w-1}(w) .
\end{aligned}
$$

Since $d^{c} \log (1 /|z-w|)=-d^{c}|z-w| /|z-w|$, we have

$$
d^{c} \lambda_{z}(w)=O_{2 n-1}|z-w|^{1-2 n} .
$$

Finally, look at $z=0$, i.e., at $a$ :

$$
\begin{aligned}
d^{c} \log (1 /|w|) \wedge\left(\omega_{n-1}(w)-v_{n-1}(w)\right)=-d^{c}|w| & \wedge\left(\omega_{n-1}(w)\right. \\
& \left.-v_{n-1}(w)\right) /|w| .
\end{aligned}
$$

Since $\omega_{n-1}(a)=v_{n-1}(a)$, this is bounded. Thus

$$
\begin{aligned}
d^{c} \lambda_{a}(w)= & {\left[(n-1) ! / 2 \pi^{n}\right] B(a, w)^{2 n-2}|w|^{2-2 n} d^{c} \log (1 /|w|) \wedge v_{n-1} } \\
& +O_{2 n-1}(w)|w|^{2-2 n} .
\end{aligned}
$$

Lemma 5. Let $f: M \rightarrow A$ be open and holomorphic, $K$ a compact subset of $M$, and $\chi$ continuous on $K$; then

$$
F(z)=\int_{K} f^{*} \lambda_{z} \wedge x
$$

exists for each $z \in A$ and is continuous.

Proof. The question is local on both $K$ and $A$, so we may assume $f(K) \subset U$ and adopt the above notation. Apply (4) and Lemma 1. q.e.d.

Lemma 6. In the same situation,

$$
G(z)=\int_{K} f^{*} d^{c} \lambda_{z} \wedge \chi
$$

exists and is continuous on $A$.

Proof. Use (7) and Lemma 1. 
3. The Residue Theorem. Let $M$ be a complex manifold of dimension $m=n+q$. Let $A$ be a compact Kähler manifold, and let $f: M \rightarrow A$ be an open holomorphic map. Let $H$ be an open subset of $M$. Let $S=\bar{H}-H$ be empty or a boundary manifold of $H$, and suppose that $\vec{H}$ is compact. Let $\psi$ be a continuous function on $\bar{H}$ satisfying a Lipschitz condition. (Then $d \psi$ exists almost everywhere.) Let $\chi$ be a differential form of bidegree $(q, q)$ and class $C^{1}$ on $M$.

Then $d^{c} f^{*} \lambda_{a} \wedge d \psi \wedge \chi$ and $d^{c} f^{*} \lambda_{a} \wedge \psi \wedge d \chi$ are integrable over $H$ for each $a \in A$.

Also let $T$ be the support of $\psi \chi$ on $S$, and assume that $T \cap f^{-1}(a)$ is a set of measure zero on $f^{-1}(a)$. Suppose that $d^{c} f^{*} \lambda_{a} \wedge \psi \chi$ is integrable on S. Then

$$
\begin{aligned}
\int_{S} d^{c} f^{*} \lambda_{a} \wedge \psi \chi= & -\int_{I} d^{c} f^{*} \lambda_{a} \wedge d \psi \wedge \chi-\int_{I I} d c f^{*} \lambda_{a} \wedge \psi \wedge d \chi \\
& +\int_{H} f^{*} \omega \wedge \psi \chi-\int_{H \cap f^{-1}(a)} \nu_{f} \psi \chi .
\end{aligned}
$$

Proof. By Stokes' Theorem,

$$
\begin{aligned}
\int_{S} \gamma_{\rho} d^{d} f^{*} \lambda_{a} \wedge \psi \chi= & -\int_{H} \gamma_{\rho} d d^{c} f^{*} \lambda_{a} \wedge d \psi \wedge \chi-\int_{I I} \gamma_{\rho} d^{c} f^{*} \lambda_{a} \wedge \psi \wedge d \chi \\
& +\int_{H} \gamma_{\rho} f^{*} \omega \wedge \psi \chi+\int_{I I} d \gamma_{\rho} \wedge a^{\prime} c f^{*} \lambda_{a} \wedge \psi \chi .
\end{aligned}
$$

We let $\rho \rightarrow 0$; from Lemma 6 we see that we need only show that the last integral has limit

$$
-\int_{H \cap f^{-1}(a)} \nu_{f} \psi \chi
$$

By (8), it is

$$
\begin{aligned}
\int_{H} d \gamma_{\rho} \wedge\left[(n-1) ! / 2 \pi^{n}\right] B(a, f)^{2 n-2}|f|^{2-2 n} & \wedge d^{c} \log (1 /|f|) \wedge v_{n-1} \wedge \psi \chi \\
& +\int_{I I} d \gamma_{\rho} f^{*} O_{2 n-1}|f|^{2-2 n} \wedge \psi \chi .
\end{aligned}
$$

By Lemma 3, the second term has limit zero. By Lemma 4, the first term has limit

$$
-\int_{I I \cap \mathcal{f}^{-1}(a)} \nu_{\rho} \psi \chi X B(a, a)^{2 n-2}
$$

but $B(a, a)=1$. q.e.d. 
Singular Stokes Theorem. Let $M, A, f, H, S$, and $\chi$ be as above. Let $\psi$ be a function of class $C^{2}$ on $\bar{H}$. Then $d^{c} f^{*} \lambda_{a} \wedge d \psi \wedge \chi, f^{*} \lambda_{a} \wedge d d^{c} \psi \wedge \chi$, and $f^{*} \lambda_{a} \wedge d^{c} \psi \wedge d \chi$ are integrable over $H$.

Assume further that $f^{*} \lambda_{a} \wedge d^{c} \psi \wedge \chi$ is integrable over $S$. Then

$$
\begin{aligned}
\int_{S} f^{*} \lambda_{a} \wedge d^{c} \psi \wedge \chi= & -\int_{H} d^{c} f^{*} \lambda_{a} \wedge d \psi \wedge \chi+\int_{H} f^{*} \lambda_{a} \wedge d d^{c} \psi \wedge \chi \\
& -\int_{I I} f^{*} \lambda_{a} \wedge d^{c} \psi \wedge d \chi .
\end{aligned}
$$

Proof. Similar to that of the Residue Theorem. Apply Lemmas 5 and 6; then consider

$$
\int_{H} d \gamma_{\rho} \wedge f^{*} \lambda_{a} \wedge d^{c} \psi \wedge \chi
$$

By (4) it is

$$
\int_{H} d \gamma_{\rho} \wedge O_{0}|f|^{2-2 n} f^{*} v_{n-1} \wedge d^{c} \psi \wedge \chi
$$

if $n>1$ and

$$
\int_{I I} d \gamma_{\rho} \wedge\left[(1 / 2 \pi) \log (1 /|f|)+O_{0}\right] \wedge d^{c} \psi \wedge \chi
$$

if $n=1$. Now apply Lemma 3. q.e.d.

The so-called Unintegrated Form of the First Main Theorem is obtained by setting $\psi=1$ and choosing $\chi$ so that $d \chi=0$ in the Residue Theorem. We can now state the:

Integrated First Main Theorem. Let $M, A, f$ and $\chi$ be as before, and assume $d \chi=0$. Let $G$ be an open subset of $M$ with compact closure $\bar{G}$. Assume that $\Gamma=\bar{G}-G$ is a boundary manifold of $G$. Let $g$ be open with $\bar{g} \subset G$, and assume that $\gamma=\bar{g}-g$ is a boundary manifold of $g$. Let $\psi$ be a continuous, real-valued function on $M$, of class $C^{2}$ on $\bar{G}-g$, such that $\psi \mid g=R$ a constant, $\psi \mid M-G=0$, and $0 \leqq \psi(z) \leqq R$ for all $z \in M$. (Such a $\psi$ satisfies a Lipschitz condition on M.) Define

$$
\begin{aligned}
& m^{*}(a)=-\int_{\Gamma} f^{*} \lambda_{a} \wedge d^{c} \psi \wedge \chi, \\
& m^{b}(a)=-\int_{\gamma} f^{*} \lambda_{a} \wedge d^{c} \psi \wedge \chi,
\end{aligned}
$$




$$
\begin{aligned}
N(a) & =\int_{f^{-1}(a)} \nu_{f} \psi \chi, \\
T & =\int_{G} f^{*} \omega \wedge \psi \chi, \\
\Delta(a) & =\int_{G} f^{*} \lambda_{a} \wedge d^{c} d \psi \wedge \chi .
\end{aligned}
$$

Then these are continuous functions on $A$, and

$$
N+m^{\#}-m^{b}=T+\Delta .
$$

Note that $N, m, m^{b}$, and $T$ are always nonnegative; but in general we have information on $\Delta$.

This theorem is obtained by applying the Residue Theorem to $G$, the Singular Stokes Theorem to $G-\bar{g}$, and eliminating the common term; see [1] for details. The continuity of $\Delta$ is a consequence of Lemma 5, and the continuity of $N$ is a theorem of Stoll [3, Theorem 3.8]. Thus $m^{\#}-m^{\text {b }}$ is continuous. Now let $h$ be a $C^{\infty}$ function with compact support in $G$ and which is 1 in a neighborhood of $\bar{g}$, and replace $\psi$ by $h \psi$. We get another FMT with different (but still continuous) $N, T$, and $\Delta$. The new $m^{\#}$ is zero but the new $m^{b}$ is the same as the old. Hence the old $m^{b}$ and $m^{\#}$ are continuous.

\section{REFERENCES}

1. J. Hirschfelder, The first main theorem of value distribution in several variables, Invent. Math. 8 (1969), 1-33.

2. W. Stoll, The multiplicity of a holomorphic map, Invent. Math. 2 (1966), 15-58.

3. - The continuity of the fiber integral, Math. Z. 95 (1967), 87-138.

4. - A general first main theorem of value distribution theory, Acta Math. 118 (1967), 111-191.

5. $\mathrm{H}$. Wu, Remarks on the first main theorem in equidistribution theory. I, II, J. Differential Geometry 2 (1968), 197-202, 369-384.

UNIVERSITY OF WASHINGTON 